\title{
New Fluorescent Probes Applicable to Aggregates of Fluorocarbon Surfactants
}

\author{
Tadahiro Ozawa, Tsuyoshi Asakawa*, Akio Ohta and Shigeyoshi Miyagishi \\ Division of Material Sciences, Graduate School of Natural Science \& Technology, Kanazawa University (Kanazawa 920-1192, JAPAN)
}

\begin{abstract}
We developed new benzofurazan (NBD) labeled probes for fluorocarbon surfactant systems. The fluorescence behavior depended on the solubilization site of the fluorescent probes in the surfactant aggregates. The NBD-labeled probes suffered virtually complete reduction in the presence of $\mathrm{Na}_{2} \mathrm{~S}_{2} \mathrm{O}_{4}$ owing to the solubilization at the surface of 2-hydroxy-1,1,2,3,3-pentahydroperfluoroundecyldiethylammonium bromide $\left(\mathrm{FC}_{8} \mathrm{DAB}\right)$ aggregates. On the other hand, $\mathrm{N}$-(3-sulfopropyl)acridinium $(\mathrm{SPA})$ in $\mathrm{FC}_{8} \mathrm{DAB}$ aggregates showed residual fluorescence in spite of $\mathrm{NaBH}_{4}$ addition. The large vesicles of $\mathrm{FC}_{8} \mathrm{DAB}$ were confirmed by DLS measurements. These facts suggest that SPA is solubilized in an inner water phase of the vesicles. The NBD labeled fluorescence probe is quite effective for the study of the aggregation behavior of fluorocarbon surfactants.
\end{abstract}

Key words: fluorescent probes, fluorocarbon surfactant, benzofurazan labeling, quenching by hydrogenation, vesicles

\section{INTRODUCTION}

The fluorescence probe method has been useful for estimating the physicochemical properties of surfactant micelles, such as critical micelle concentration (CMC), the degree of micellar ionization, micellar micropolarity, and micellar microviscosity ${ }^{1-7}$. We found that halide-sensitive 6-methoxy-N-(3-sulfopropyl)quinolinium (SPQ) fluorescence probe method was effective for the simultaneous determination of CMC and the degree of micellar ionization by fluorescence quenching behavior ${ }^{2}$. Pyrene fluorescence probe method has been widely used to estimate $\mathrm{CMC}$ and micellar micropolarity ${ }^{4,5}$. However, we previously reported that the $I_{1} / I_{3}$ ratio of pyrene was not changed by the micelle formation of cationic fluorocarbon surfactant because fluorocarbon chain has a low solubilization power toward pyrene $^{8,9)}$. A suitable fluorescent probe is required for estimating the micellar micropolarity of fluorosurfactants. Over the past a few decades, NBD fluorescence-labeled phospholipids have been developed to evaluated the properties of vesicles in order to gain the insight into the phospholipid vesicles ${ }^{10-12)}$. NBD-labeled lipids have been used to estimate the aggregation behavior of vesicles. In addition, the NBD fluorescent probe has unique property that it becomes nonfluorescent in the presence of $\mathrm{Na}_{2} \mathrm{~S}_{2} \mathrm{O}_{4}$, the reducing agent; this property enables us to provide the information of solubilization site of the probe molecule in vesicles.

The solubilization of fluorescent probes in micelles has been investigated by several methods ${ }^{10-13)}$. We confirmed that the gel filtration method was also useful to reveal the partition of fluorescent probes between the bulk and micellar phases ${ }^{2}$. The hydrophilic SPQ tend to partition in bulk phase without being trapped in micelles, whereas the hydrophobic pyrene is almost completely solubilized in tetradecyltrimethylammonium chloride (TTAC) micelles. We also confirmed that the fluorescence of SPQ was remarkably reduced by the hydrogenation with $\mathrm{NaBH}_{4}$. The solubilization site of SPQ probes in micelles could be investigated from the variation in fluorescence intensities by the hydrogenation.

In this paper, the aggregation behavior of $\mathrm{FC}_{8} \mathrm{DAB}$ was investigated by the characteristic fluorescence of new amphiphilic NBD labeled probes, in comparison with Ndodecyl-N-methylephedrinium bromide (DMEB). The solubilization site of NBD labeled probes in surfactant aggregates was examined by the reduction of fluorophore using $\mathrm{Na}_{2} \mathrm{~S}_{2} \mathrm{O}_{4}$, in comparison with SPA reduced by $\mathrm{NaBH}_{4}$. The solubilization of probes was also examined by gel filtration method. The size of $\mathrm{FC}_{8} \mathrm{DAB}$ aggregates was examined by the measurements of dynamic light scattering.

\footnotetext{
*Correspondence to: Tsuyoshi Asakawa, Division of Material Sciences, Graduate School of Natural Science \& Technology, Kanazawa University, Kanazawa 920-1192, JAPAN

E-mail: asakawa@t.kanazawa-u.ac.jp

Accepted August 3, 2007 (received for review June 5, 2007)
}

Journal of Oleo Science ISSN 1345-8957 print / ISSN 1347-3352 online

http://jos.jstage.jst.go.jp/en/ 


\section{T. Ozawa, T. Asakawa, A. Ohta and S. Miyagishi}

\section{EXPERIMENTAL}

\subsection{Materials}

2-Hydroxy-1,1,2,3,3-pentahydroperfluoroundecyl diethylammonium bromide $\left(\mathrm{FC}_{8} \mathrm{DAB}\right)$ was prepared as reported previously $^{8)}$. N-Dodecyl-N-methylephedrinium bromide (DMEB, Aldrich) and N-(3-sulfopropyl)acridinium (SPA, Molecular Probes, Inc.) and pyrene (Aldrich) were used as received.

The surfactant was labeled with 4-chloro-7-nitrobenzo-2oxa-1,3-diazol (NBD-Cl, Wako Pure Chem. Co.) by the incubation at $40^{\circ} \mathrm{C}$ in micellar aqueous solution, that is, the solution of $0.25 \mathrm{mM} \mathrm{NBD-Cl}$ in $10 \mathrm{mM}$ surfactant was stirred for 4 days. The absorption band around $468 \mathrm{~nm}$ appeared with the introduction of NBD into hydroxyl groups of $\mathrm{FC}_{8} \mathrm{DAB}$. The NBD labeled probes were confirmed by HPLC analysis using TSKgel ODS-100V column (TOSOH Co.) with the elution of methanol/0.4 M NaCl aqueous solution $(85 / 15, \mathrm{v} / \mathrm{v})$.

\subsection{Measurements}

\subsubsection{Fluorescence measurements}

The aqueous solution of $5.0 \times 10^{-7} \mathrm{M}$ SPA was made up in doubly distilled water. The fluorescence intensities were measured at $485 \mathrm{~nm}$ after the excitation at $417 \mathrm{~nm}$ using a Hitachi F-2000 spectrophotometer. The fluorescence intensity in water without quencher $\left(I_{0}\right)$ was used as a standard. The fluorescence spectra of $1.0 \times 10^{-7} \mathrm{M}$ pyrene were measured in the range from $350 \mathrm{~nm}$ to $450 \mathrm{~nm}$ after the excitation at $335 \mathrm{~nm}$ using a Hitachi F-3010 spectrometer. The ratio of first and third peaks has a value of 1.88 in water. The fluorescence intensities of $1.0 \times 10^{-5} \mathrm{M} \mathrm{FC}_{8} \mathrm{NBD}$ and DMENBD aqueous solutions were measured at $540 \mathrm{~nm}$ after the excitation at $468 \mathrm{~nm}$ using a Hitachi F-2000 spectrophotometer.

The hydrogenation of $5.0 \times 10^{-7} \mathrm{M}$ SPA was performed in a quartz cell by the addition of $5 \mu \mathrm{L}$ of $0.1 \mathrm{M} \mathrm{NaBH}_{4}$ aqueous solution ${ }^{13,14)}$. The reduction of $1.0 \times 10^{-5} \mathrm{M} \mathrm{FC}_{8} \mathrm{NBD}$ was achieved by the addition of $10 \mu \mathrm{L}$ of $1 \mathrm{M} \mathrm{Na}_{2} \mathrm{~S}_{2} \mathrm{O}_{4}$ aqueous solution. The fluorescence spectra were recorded by using a Perkin-Elmer LS-55 spectrophotometer. All experiments were performed at $25^{\circ} \mathrm{C}$.

\subsubsection{Gel filtration}

Sephadex G-25, a cross-linked dextran gel, was swelled in distilled water. The gel was packed into XK-16/20 column at the gel height $12 \mathrm{~cm}$ (Pharmacia Fine Chem.). The void volume of the column was checked by using Blue Dextran 2000 (Pharmacia Fine Chem.). The column was equilibrated with $10^{-7} \mathrm{M}$ SPA aqueous solution without surfactant at $25^{\circ} \mathrm{C}$. Then $20 \mathrm{~mL}$ of surfactant solution containing $10^{-7} \mathrm{M}$ SPA was eluted with $10^{-7} \mathrm{M}$ SPA eluate at a flow rate of $24 \mathrm{~mL} / \mathrm{h}$. The fluorescence intensity of SPA was monitored by a TOSHO FS-8020 fluorescence detector. The elution of surfactant was detected by a HORIBA DS12 conductivity meter using a flowcell.

\subsubsection{DLS measurements}

Dynamic light scattering apparatus (Malvern HPP-5001) was used to determine the size of surfactant aggregates at $25^{\circ} \mathrm{C}$. Samples were repeatedly filtered through a membrane filter with $1 \mu \mathrm{m}$ pores as pretreatment of measurements.

\section{RESULTS AND DISCUSSION}

\subsection{Fluorescence probe methods}

The fluorescence intensity ratio of the first $\left(I_{1}\right)$ and third $\left(I_{3}\right)$ vibronic peaks of pyrene has been used for the determination of $\mathrm{CMC}$ and micellar micropolarity ${ }^{4)}$. Figure 1 shows the variation of pyrene fluorescence intensity ratios $\left(I_{1} / I_{3}\right)$ with the surfactant concentration. The decrease in the $I_{1} / I_{3}$ values is ascribed to the solubilization of pyrene into the micelles. The considerable decrease in the $I_{1} / I_{3}$ value from 1.88 to 1.35 was observed with the formation of DMEB micelles, suggesting the micellar micropolarity similar to the polarity of methanol $\left(I_{1} / I_{3}=1.30\right.$ in methanol). The inflection point in Fig. 1 was estimated to be $3.9 \mathrm{mM}$, which is consistent with the CMC $(3.9 \mathrm{mM})$ of DMEB reported by S. Roy et $a l^{15)}$.

In contrast, no inflection point was observed for $\mathrm{FC}_{8} \mathrm{DAB}$ because the location of pyrene would be close to the micelle surface owing to the low affinity of pyrene for fluorocarbon chain. The values of $I_{1} / I_{3}$ were virtually identical to that of water even at the concentration above the $\mathrm{CMC}$ of $\mathrm{FC}_{8} \mathrm{DAB}^{8}$. Thus $\mathrm{CMC}$ and micellar micropolarity of $\mathrm{FC}_{8} \mathrm{DAB}$ cannot be determined by using pyrene fluorescence intensity ratios. A suitable fluorescent probe is required for the evaluation of micellar micropolarity of $\mathrm{FC}_{8} \mathrm{DAB}$.

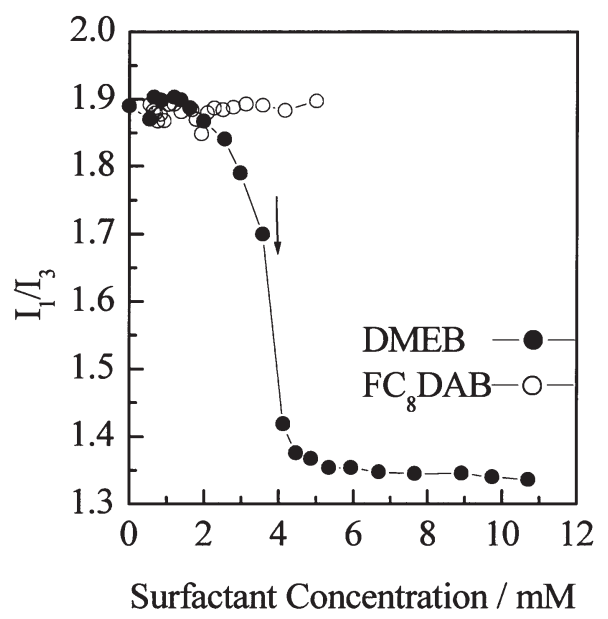

Fig. 1 Pyrene Fluorescence Ratios $I_{1} / I_{3}$ vs. Surfactant Concentration. 
4-Chloro-7-nitrobenzo-2-oxa-1,3-diazol (NBD-Cl) has been used for detecting small quantities of amines and amino acids ${ }^{16,17}$. NBD-Cl is non-fluorescent compound, while NBD labeled derivatives gave strong fluorescence resulting from the reaction of $\mathrm{NBD}-\mathrm{Cl}$ and amino groups. The reaction was reported to occur at neutral $\mathrm{pH}$ even at room temperature. We aimed to label $\mathrm{FC}_{8} \mathrm{DAB}$ and $\mathrm{DMEB}$ molecules with NBD-Cl at the hydroxyl group of the surfactants. The fluorescent NBD labeled probes would be useful for evaluating the microenvironment of surfactant aggregates; they have an advantage that the location in the aggregates probed by these molecules is rather specified, because the probe molecules themselves are also surfactants. The chemical structures of NBD labeled surfactant are shown in Scheme 1. The distinct absorption peak appeared at $468 \mathrm{~nm}$ as a result of the introduction of NBD$\mathrm{Cl}$ to $\mathrm{FC}_{8} \mathrm{DAB}$ and DMEB surfactants, as shown in Fig. 2. $\mathrm{FC}_{8} \mathrm{NBD}$ and DMENBD in aqueous solution gave a broad fluorescence emission peak centered at $540 \mathrm{~nm}$ by the excitation at $468 \mathrm{~nm}$. Figure 3 shows NBD fluorescence intensity ratios $\left(I / I_{0}\right)$ at $468 \mathrm{~nm}$ plotted against the surfactant concentration. The fluorescence intensity in water, $I_{0}$, was used as a standard. The fluorescence intensities increased with increasing surfactant concentration without spectral shifts. The abrupt increase in fluorescence intensity may be due to the solubilization of NBD-labeled probes into the micelles. The CMC of the surfactants can be determined from the inflection points in the fluorescence intensity vs. concentration plots, as indicated by the arrows in Fig. 3 . The $\mathrm{CMC}$ values thus obtained for $\mathrm{FC}_{8} \mathrm{DAB}$ and $\mathrm{DMEB}$

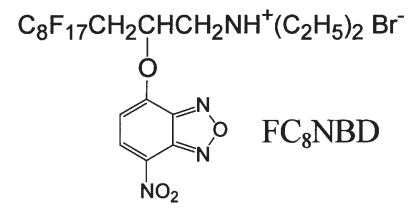

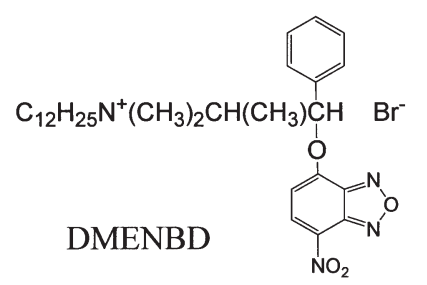

Scheme 1 Chemical Structures of O-(4-(7-nitorobenzofurazan))-(2-oxy-1,1,2,3,3-pentahydroperfluoro-undecyldiethylammonium Bromide $\left(\mathrm{FC}_{8} \mathrm{NBD}\right)$ and N-dodecyl-N-(O- $(4-$ (7-nitoro-benzofurazan))-(2-oxy-1-methyl2phenylethyl))-N,N-dimethyammonium Bromide (DMENBD). were 1.8 and $3.9 \mathrm{mM}$, respectively. The fluorescence intensity became almost constant at higher concentrations above CMC. The $I / I_{0}$ values above $\mathrm{CMC}$ gave remarkable difference between $\mathrm{FC}_{8} \mathrm{DAB}$ and $\mathrm{DMEB}$. The difference could be ascribed to the solubilization site of the probes. The solubilization site of NBD group would be located at $\mathrm{FC}_{8} \mathrm{DAB}$ micellar surface since the fluorocarbon chain is repulsive to organics such as NBD group. In any event, $\mathrm{FC}_{8} \mathrm{NBD}$ probe is useful to determine the $\mathrm{CMC}$ of $\mathrm{FC}_{8} \mathrm{DAB}$.

\subsection{Determination of CMC using various fluorescent probes}

The solubility of SPA in water is $7 \times 10^{-3} \mathrm{M}^{18)}$, which is about 10,000 times larger than that of pyrene $\left(6.7 \times 10^{-7}\right.$ $M{ }^{19}$. Thus SPA will tend to partition in aqueous bulk

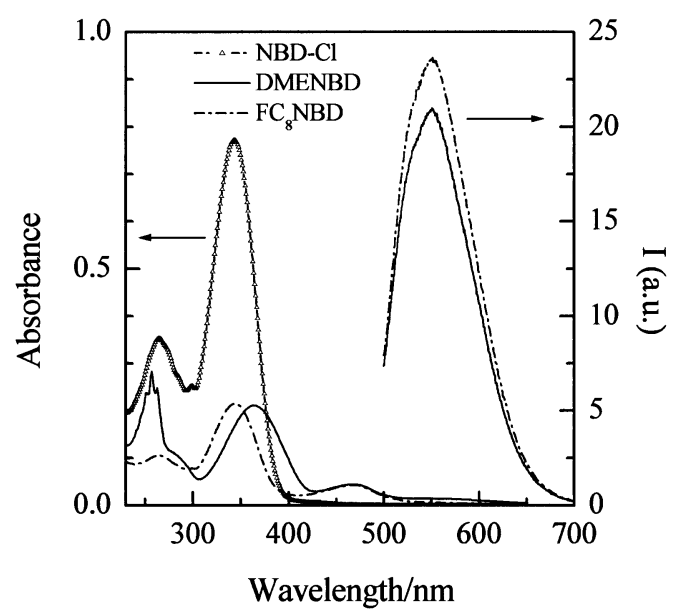

Fig. 2 Absorption and Emission Spectra of NBD Derivatives in Aqueous Solution.

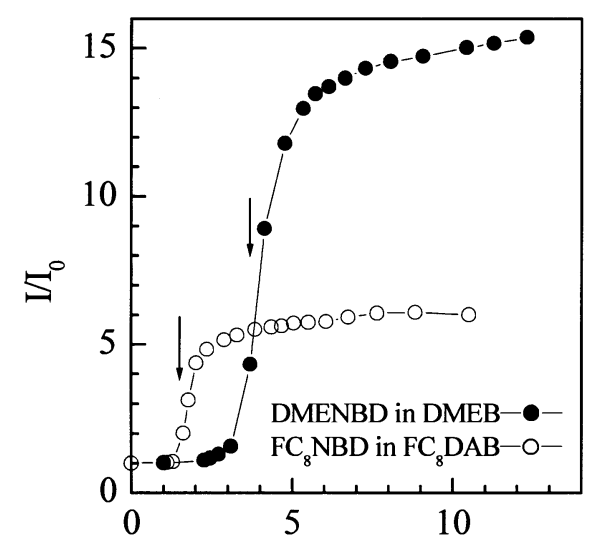

Surfactant Concentration/mM

Fig. 3 Fluorescence Intensity Ratios $I / I_{0}$ of DMENBD and $\mathrm{FC}_{8} \mathrm{NBD}$ in Surfactant Aqueous Solution. 


\section{T. Ozawa, T. Asakawa, A. Ohta and S. Miyagishi}

phase, whereas pyrene is solubilized into micellar phase. The SPA fluorescence is quenched by halide ions in aqueous solutions ${ }^{20,21)}$. The halide sensitive fluorescence probe, SPA, provides a direct approach for measurement of bromide concentrations dissociated from the surfactant. When quenching occurs by the collision between SPA and bromide ions, the variation of fluorescence intensity are related to the concentration of bromide ions by the SternVolmer equation.

$$
I_{0} / I=1+K_{S V}[\mathrm{Br}]
$$

where $I_{0}$ and $I$ are the fluorescence intensities in the absence and presence of quencher, respectively, and $K_{S V}$ is the Stern-Volmer constant. The fluorescence of SPA was quenched with a linear Stern-Volmer relation for sodium bromide system as shown in Fig. 4. The $K_{S V}$ value of 345 $\mathrm{M}^{-1}$ for sodium bromide system gave excellent agreement with those of surfactants below CMC. The Stern-Volmer plot gave a distinct break at the $\mathrm{CMC}$ owing to the binding of bromide counterion toward the micelles. The CMC values for $\mathrm{FC}_{8} \mathrm{DAB}$ and $\mathrm{DMEB}$ were obtained as 1.8 and 4.3 $\mathrm{mM}$, respectively. The degree of micellar counterion dissociation, $\alpha$, was taken as the ratio of the values of $K_{S V}$ above and below the CMC. The CMC values determined by SPA, Pyrene and NBD-labeled probes were summarized in Table 1. The $\mathrm{CMC}$ values of $\mathrm{FC}_{8} \mathrm{DAB}$ are in fair agreement with the experimental values determined by the conductivity (1.9 $\mathrm{mM}$ from our experimental data) and SPQ fluorescence quenching method $(1.85 \mathrm{mM})^{8}$. The $\alpha$ value of DMEB (0.28) was much larger than 0.18 of dodecyltrimethylammonium bromide micelle owing to the bulky ephedrine group ${ }^{15)}$. The $I / I_{0}$ value for $\mathrm{FC}_{8} \mathrm{NBD}$ in methanol was in accordance with that for DMENBD in ethanol according to our experimental data $\left(I / I_{0}=12\right.$ for both cases), while in micellar systems, the $I / I_{0}=6$ for $\mathrm{FC}_{8} \mathrm{DAB}$ was considerably lower than $I / I_{0}=15$ for DMEB. The significant difference for these NBD labeled probes would be ascribed to the difference in the solubilization site of NBD groups in micelles. NBD group of DMENBD would be buried in DMEB micellar core, while NBD group of $\mathrm{FC}_{8} \mathrm{NBD}$ would be present at the micellar surface avoiding the contact with fluorocarbon chains.

\subsection{Gel filtration and DLS}

Gel filtration of surfactant aqueous solution was exam- ined to evaluate the solubilization of probes in micelles. The gel filtration method can separate the monomers and/or probes in the bulk water phase from micellar solutions $^{2,23,24)}$. The hydrophilic 6-methoxy-N-(3-sulfopropyl) quinorinium $(\mathrm{SPQ})$ was completely separated from the cationic micelles, while the hydrophobic pyrene eluted together with micelles ${ }^{2)}$. Thus the elution behavior will depend on the solubilization mode of probes in micelles.

When the Sephadex G-25 column for gel filtration was equilibrated with the enough volumes of micellar solution, the monomers in the bulk water phase can be separated from micellar solution in tail region because the micelles are eluated in preference to monomers. This tail analysis method was examined for $\mathrm{FC}_{8} \mathrm{DAB}$ solution in the presence of $\mathrm{SPA}$ or $\mathrm{FC}_{8} \mathrm{NBD}$ probes. Figure 5 shows the elution profiles of surfactant and probes in $10 \mathrm{mM} \mathrm{FC}_{8} \mathrm{DAB}$ aqueous solution. The electric conductivity curve shows that the micellar region corresponds to the elution volume of $7 \mathrm{~mL}$ to $12 \mathrm{~mL}$, whereas the monomer region corresponds to the elution volume of $12 \mathrm{~mL}$ to $17 \mathrm{~mL}$. The SPA fluorescence intensity remained constant at $I=65$, while it decreased to $I=40$ associated with the elution of micelles. The SPA fluorescence intensity for the monomer region increased slightly to give $I=43$. The SPA fluorescence intensities can be used to determine the concentration of free bromide ions in equilibrium with surfactants after gel filtration. These experiments gave the fluorescence intensity ratios

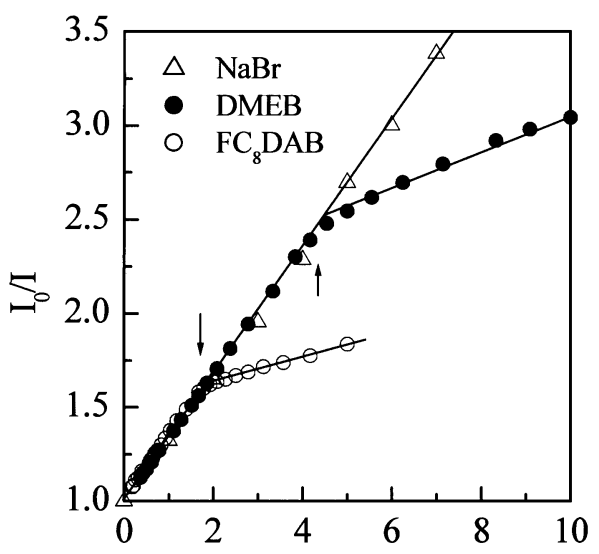

Surfactant Concentration /mM

Fig. 4 Stern-Volmer Plots for Quenching of SPA Fluorescence.

Table 1 Micellar Solution Properties Determined by Fluorescent Probes at $25^{\circ} \mathrm{C}$.

\begin{tabular}{|c|c|c|c|c|c|c|}
\hline \multirow{2}{*}{ Surfactant } & \multicolumn{2}{|c|}{ NBD } & \multicolumn{2}{|c|}{ pyrene } & \multicolumn{2}{|c|}{ SPA } \\
\hline & $\mathrm{CMC} / \mathrm{mM}$ & $I / I_{0}$ & $\mathrm{CMC} / \mathrm{mM}$ & $I_{1} / I_{3}$ & $\mathrm{CMC} / \mathrm{mM}$ & $\alpha$ \\
\hline $\mathrm{FC}_{8} \mathrm{DAB}$ & 1.8 & 6 & - & 1.90 & 1.8 & 0.19 \\
\hline DMEB & 3.9 & 15 & 3.9 & 1.35 & 4.3 & 0.28 \\
\hline
\end{tabular}




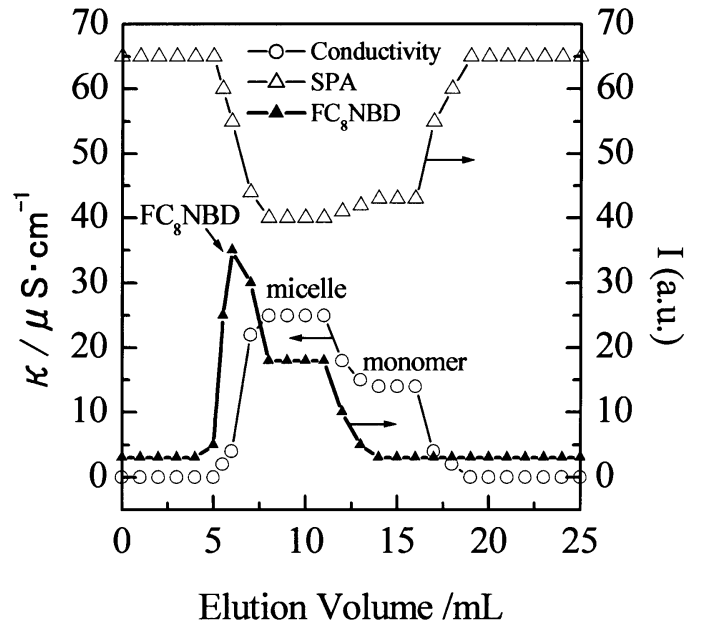

Fig. 5 Elution Curves of $10 \mathrm{mM} \mathrm{FC} \mathrm{C}_{8} \mathrm{DAB}$ Containing Fluorescence Probe Using Sephadex G-25.

of SPA for eluted solutions as shown in Fig. 6. The SternVolmer plot gave a distinct break at the CMC owing to the binding of bromide counterion to the micelles in the same manner as Fig. 4. The $\mathrm{CMC}$ values obtained for $\mathrm{FC}_{8} \mathrm{DAB}$ and DMEB were 2.1 and $4.2 \mathrm{mM}$, respectively. This figure also indicated that the concentration of free bromide ion being in equilibrium with eluted monomer was almost constant at the concentration above the CMC.

As shown in Fig. 5, the abrupt increase in fluorescence intensity of $\mathrm{FC}_{8} \mathrm{NBD}$ was observed in frontal region. This elution would correspond to the large aggregates such as vesicles containing $\mathrm{FC}_{8} \mathrm{NBD}$ probes. The coexistence of larger aggregates with micelles in $10 \mathrm{mM} \mathrm{FC}_{8} \mathrm{DAB}$ was suggested by the elution profiles of $\mathrm{FC}_{8} \mathrm{NBD}$ probe. The diameter of $\mathrm{FC}_{8} \mathrm{DAB}$ aggregates was determined by using dynamic light scattering as shown in Fig. 7. The spherical micelles about $2 \mathrm{~nm}$ coexisted with large aggregates about $300 \mathrm{~nm}$ in $10 \mathrm{mM} \mathrm{FC}_{8} \mathrm{DAB}$. The coexistence of micelles and the vesicles was detected for 2-hydroxy-1,1,2,3,3-pentahydroperfluoroundecyldiethyl ammonium chloride $\left(\mathrm{FC}_{8} \mathrm{DAC}\right)$ aqueous solutions in the presence of $\mathrm{NaCl}^{22)} \cdot \mathrm{FC}_{8} \mathrm{DAB}$ would be prone to self-assemble into the vesicles without the addition of salt owing to the preferred binding of bromide counterions for the surfactant aggregates.

\subsection{Quenching of fluorescence probes by the reduction}

We aimed to confirm the presence of vesicles, i.e., inner aqueous phases, by using the change in SPA fluorescence spectra caused by hydrogenation. The $\mathrm{NaBH}_{4}$ reduction of 6-methoxy-N-(3-sulfopropyl)quinorinium (SPQ) probes has been reported as a method to judge whether the inner aqueous phase is present or not ${ }^{16,20)}$. The SPQ dissolved in micellar aqueous solution underwent instantaneous reduc-

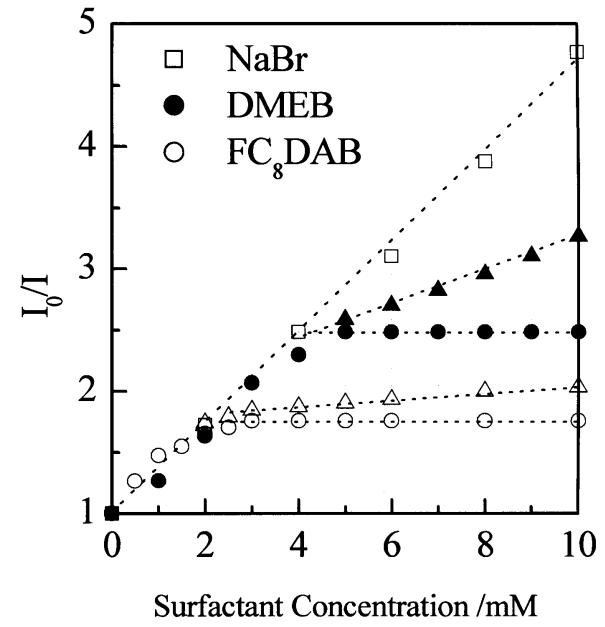

Fig. 6 Stern-Volmer Plots for Quenching of SPA Fluorescence. (circles): Eluted Monomer Solution, (triangles): Eluted Micelle Solution.

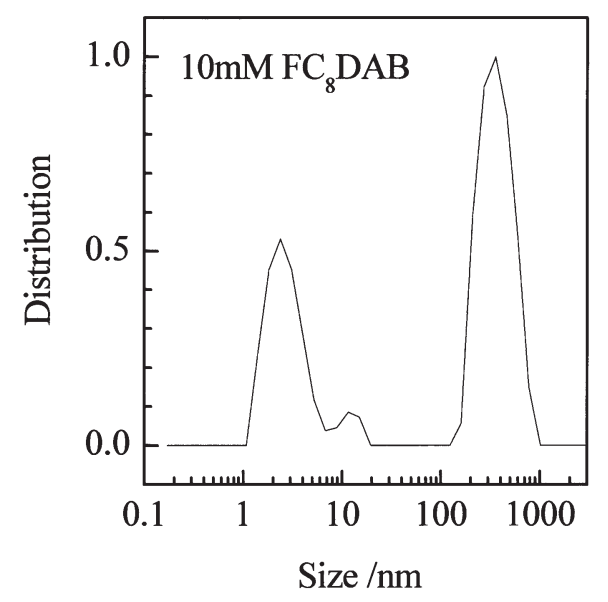

Fig. 7 Diameters of Surfactant Aggregates in $10 \mathrm{mM} \mathrm{FC}_{8} \mathrm{DAB}$ Aqueous Solution.

tion with the addition of $\mathrm{NaBH}_{4}$, while $\mathrm{SPQ}$ in vesicles was hardly reduced owing to the solubilization into an inner aqueous phase ${ }^{20}$. We confirmed that the hydrogenated SPA by $\mathrm{NaBH}_{4}$ gave a remarkable decrease in fluorescence intensity in aqueous solution. The addition of hydrogen to the 9th position of acridine backbone by using $\mathrm{NaBH}_{4}$ aqueous solution was reported ${ }^{25}$. Significant decrease in fluorescence intensity suggested that the hydrogenated SPA is non-fluorescent. Figure 8 shows the fluorescence spectral change of $10^{-7} \mathrm{M}$ SPA in aqueous solutions by the addition of $\mathrm{NaBH}_{4}$. The SPA probe was instantly reduced by $\mathrm{NaBH}_{4}$ in aqueous solution, resulting in the significant decrease of fluorescence. However, the considerable fluorescence intensity of SPA remained when $\mathrm{NaBH}_{4}$ was added to 10 


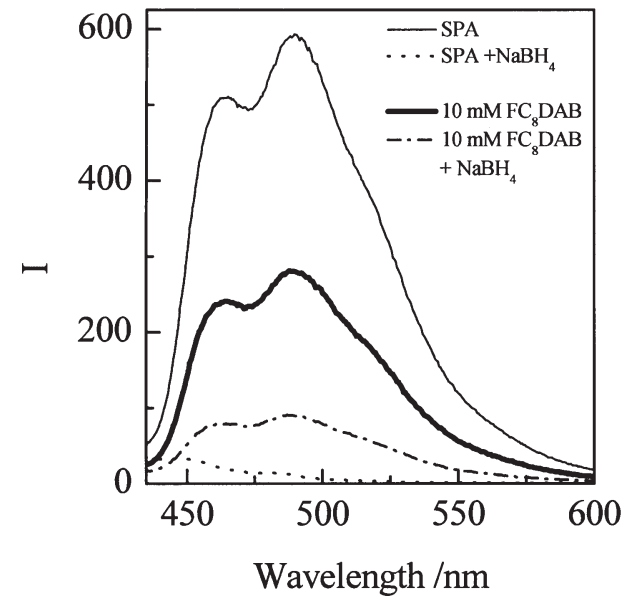

Fig. 8 Spectral Changes in SPA Fluorescence by Addition of $\mathrm{NaBH}_{4}$ in Aqueous Solutions.

Solid lines indicate SPA fluorescence in water and $10 \mathrm{mM} \mathrm{FC}_{8} \mathrm{DAB}$ aqueous solutions, respectively. Dotted line indicates hydrogenated SPA in water after the addition of $\mathrm{NaBH}_{4}$. Dot-dashed line indicates fluorescence spectra in $10 \mathrm{mM} \mathrm{FC}_{8} \mathrm{DAB}$ aqueous solutions after the addition of $\mathrm{NaBH}_{4}$.

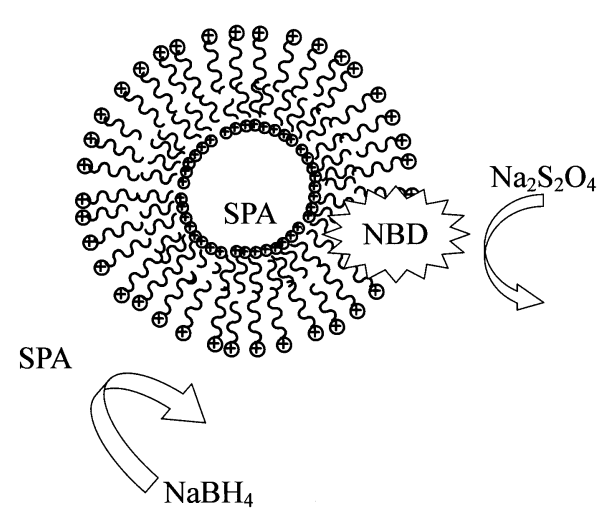

Scheme 2 Schematic Model for Fluorescence Quenching of SPA and NBD Labeled Probes Caused by the Reduction in $\mathrm{FC}_{8} \mathrm{DAB}$ Vesicle Systems.

$\mathrm{mM} \mathrm{FC} \mathrm{C}_{8} \mathrm{DAB}$ systems. This suggests the existence of the inner aqueous phase containing SPA. That is to say, the remained fluorescence intensity would be attributed to the SPA dissolved in the inner aqueous phase of vesicles as shown in Scheme 2.

The reduction of NBD labeled probes has been reported as a method to judge the solubilization site of probe molecules between inner and outer leaflets of the phospholipid vesicle membranes. It has been reported that the

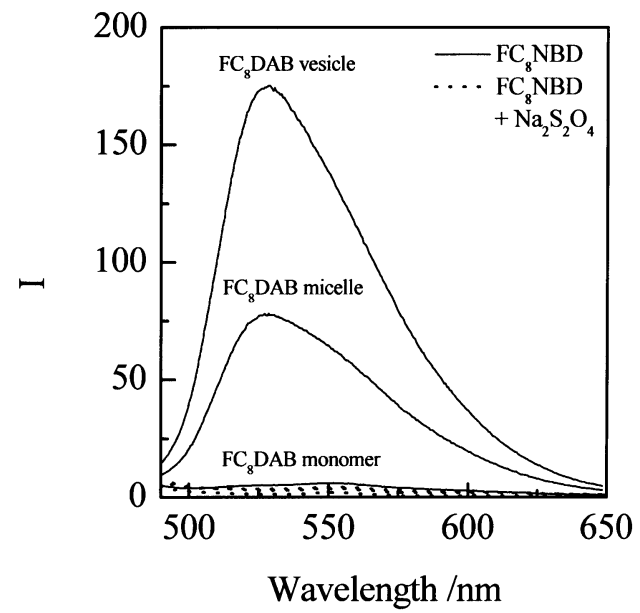

Fig. 9 Effect of $\mathrm{Na}_{2} \mathrm{~S}_{2} \mathrm{O}_{4}$ Addition on $\mathrm{FC}_{8} \mathrm{NBD}$ Fluorescence Spectra in $\mathrm{FC}_{8} \mathrm{DAB}$ Aqueous Solutions Batched off by the Gel Filtration.

nitro group of the NBD probe was reduced to an amino group by the addition of $\mathrm{Na}_{2} \mathrm{~S}_{2} \mathrm{O}_{4}{ }^{11}$. The solubilization site of $\mathrm{FC}_{8} \mathrm{NBD}$ probes in $\mathrm{FC}_{8} \mathrm{DAB}$ aggregates was also examined by the reduction behavior of NBD fluorescence with the addition of $\mathrm{Na}_{2} \mathrm{~S}_{2} \mathrm{O}_{4}$. That is, the variation in $\mathrm{FC}_{8} \mathrm{NBD}$ fluorescence spectra would be used to evaluate the solubilization site of the probe in $\mathrm{FC}_{8} \mathrm{DAB}$ aggregates. Figure 9 shows the fluorescence spectral change of $10^{-7} \mathrm{M} \mathrm{FC}_{8} \mathrm{NBD}$ in $\mathrm{FC}_{8} \mathrm{DAB}$ solutions by the addition of $\mathrm{Na}_{2} \mathrm{~S}_{2} \mathrm{O}_{4}$. The fluorescence intensity of $\mathrm{FC}_{8} \mathrm{NBD}$ for $\mathrm{FC}_{8} \mathrm{DAB}$ vesicles was larger than that of micelles batched off by the gel filtration, but it significantly decreased by the addition of $\mathrm{Na}_{2} \mathrm{~S}_{2} \mathrm{O}_{4}$ as well as micelles. This suggests that $\mathrm{FC}_{8} \mathrm{NBD}$ probes would be located at the surface of $\mathrm{FC}_{8} \mathrm{DAB}$ vesicles.

\section{CONCLUSION}

We developed the new NBD labeled fluorescence probes in order to estimate the aqueous solution properties of fluorosurfactants. It was demonstrated that $\mathrm{FC}_{8} \mathrm{NBD}$ is a quite effective probe to the $\mathrm{CMC}$ determination for $\mathrm{FC}_{8} \mathrm{DAB}$ in contrast to pyrene. The fluorescent NBD labeled probes would be suitable for evaluating the microenvironment of fluorocarbon aggregates, since due to their characteristic structure that a fluorophore is attached to the fluorocarbon surfactants, the perturbation caused by the addition of the probes would be suppressed at low level, and also, the location probed in the aggregates can be rather specified. 


\section{References}

1. Singer, L.A. Fluorescence probes of micellar systemsAn overview, Solution Behavior of surfactants, Plemnum, New York, pp.73-112 (1982).

2. Asakawa, T.; Kubode, H.; Ozawa, T.; Ohta, A.; Miyagishi, S. Micellar counterion binding probed by fluorescence quenching of 6-methoxy-N-(3-sulfopropyl)quinolinium. J. Oleo Sci. 54, 545-552 (2005).

3. Ceapkiewicz, J.; Dlugolecka, M.; Tutaj, B. 10-Methylacridinium ion as a fluorimetric probe measuring the activity of halide anions in aqueous solutions of cationic surfactants. J. Colloid Interface Sci. 276, 227-230 (2004).

4. Kalyanasundaram, K. Pyrene fluorescence as a probe of fluorocarbon micelles and their mixed micelles with hydrocarbon surfactants. Langmuir 4, 942-945 (1988).

5. Ananthapadmanabhan, K.P.; Goddard, E.D.; Turro, N. J.; Kuo, P.L. Fluorescence probes for critical micelle concentration determination. Langmuir 1, 352-355 (1985).

6. Zachariasse, K.A. Intramolecular excimer formation with diarylalkanes as a microfluidity probe for sodium dodecyl sulphate micelles. Chem. Phys. Lett. 57, 429432 (1978).

7. Miyagishi, S.; Suzuki, H.; Asakawa, T. Microviscosity and aggregation number of potassium $N$-acylalaninate micelles in potassium chloride solution. Langmuir 12, 2900-2905 (1996).

8. Ozawa, T.; Asakawa, T.; Garamus, V.M.; Ohta, A.; Miyagishi, S. Effect of $\mathrm{D}_{2} \mathrm{O}$ solvent on the micellization behavior of 2-hydroxy-1,1,2,3,3-pentahydroperfluoroundecyl diethylammonium halides. J. Oleo Sci. 54, 585-588 (2005).

9. Asakawa, T.; Amada, K.; Miyagishi, S. Micellar immiscibility of lithium 1,1,2,2-tetrahydroheptadecafluorodecyl sulfate and lithium tetradecyl sulfate mixture. Langmuir 13, 4569-4573 (1997).

10. Galinier, F.; Bertorelle, F.; Frey-Forgues, S. Spectrophotometric study of the incorporation of NBD probes in micelles: Is a long alkyl chain on the fluorophore an advantage? C. R. Acad. Sci. Paris, Chemie/ Chemistry 4, 941-950 (2001).

11. McIntyre, J.C.; Sleight, R.G. Fluorescence assay for phospholipid membrane asymmetry. Biochemistry 30, 11819-11827 (1991).

12. Heureux, G.P.L.; Fragata, M. Micropolarities of lipid bilayers and micelles: 5 . Localization of pyrene in small unilamellar phosphatidylcholine vesicles, Biophysical Chemistry 30, 293-301 (1988).

13. Kuwamoto, K.; Asakawa, T.; Ohta, A.; Miyagishi, S.
Degree of micelle ionization and micellar growth for gemini surfactants detected by 6-methoxy- $N$-(3-sulfopropyl)quinolinium fluorescence quenching. Langmuir 21, 7691-7695 (2005).

14. Biwersi, J.; Verkman, A.V. Cell-permeable fluorescent indicator for cytosolic chloride. Biochemistry 30, 78797883 (1991).

15. Roy, S.; Khatua, D.; Dey, J. Giant vesicles of a singletailed chiral cationic surfactant, (1R,2S)-(-)-N-dodecyl- $N$-methylephedrinium bromide, in water. J. Colloid Interface Sci. 292, 255-264 (2005).

16. Uchiyama, S.; Santa, T., Okiyama, N.; Fukushima, T.; Imai, K. Fluorogenic and fluorescent labeling reagents with a benzofurazan skeleton. Biomed. Chromatogr. 15, 295-318 (2001).

17. Numasawa, Y.; Okabe, K.; Uchiyama, S.; Imai, K. Fluorescence characteristics of ionic benzofurazans, 7 -substituted-2,1,3-benzoxadiazole-4-sulfonates. Dyes and Pigments 67, 189-195 (2005).

18. Kraft, R.; Illsley, N.P.; Tseng, H.C.; Verkman, A.S. Structure-activity relationships of chloride-sensitive fluorescent indicators for biological application. Anal. Biochem. 169, 142-150 (1988).

19. Davis, W.W.; Krahl, M.E., Clowes, G.H.A. Solubility of carcinogenic and related hydrocarbons in water. $J$. Am. Chem. Soc. 64, 108-110 (1942).

20. Ozawa, T.; Asakawa, T.; Ohta, A.; Miyagishi, S. Halidesensitive fluorescent probes for self-assembly of novel cationic fluorosurfactants. J. Oleo Sci. 54, 135-142 (2005).

21. Asakawa, T.; Iriyama, K.; Ohta, A.; Miyagishi, S. Micellar counterion binding for alkylpyridinium iodides probed by $\mathrm{N}$-sulfopropylacridinium fluorescence quenching. J. Oleo Sci. 53, 445-451 (2004).

22. Wang, K.; Karlson, G.; Almgren, M., Asakawa, T. Aggregation behavior of cationic fluorosurfactants in water and salt solutions. A cryoTEM survey. J. Phys. Chem. B 103, 9237-9246 (1999).

23. Herries, D.G.; Bishop, W. Richards, F.M.; The partitioning of solutes between micellar and aqueous phases: Measurement by gel Filtration and effect on the kinetics of some bimolecular reactions 1. J. Phys. Chem. 68, 1842-1852 (1964).

24. Tokiwa, F.; Ohki, K.; Kokubo, I. Gel filtration of surfactants on Sephadex. Bull. Chem. Soc. Jpn. 41, 22852288 (1968).

25. Keay, J.G. The reduction of nitrogen heterocycles with complex metal hydrides. Adv. Heterocycl. Chem. 39, 177 (1896). 